EXTENDED REPORT

\title{
In vivo cartilage deformation after different types of activity and its dependence on physical training status
}

\author{
F Eckstein, B Lemberger, C Gratzke, M Hudelmaier, C Glaser, K-H Englmeier, M Reiser
}

Ann Rheum Dis 2005;64:291-295. doi: 10.1136/ard.2004.022400

See end of article for authors' affiliations ....................

Correspondence to: Dr P D med. Felix Eckstein, Institut für Anatomie und Muskuloskelettale Forschung, Paracelsus Medizinische Privatuniversität, Strubergasse 21, A-5020 Salzburg, Austria; eckstein@anat.med. uni-muenchen.de

Accepted 16 May 2004
Background: Knowledge of the deformational behaviour of articular cartilage in vivo is required to understand the pathogenesis of osteoarthritis and the mechanical target environment of prospective cartilage transplant recipients.

Objectives: To study the in vivo deformational behaviour of patellar and femorotibial cartilage for different types of physiological activities; and to test the hypothesis that in vivo deformation of cartilage is modified by intense physical exercise.

Methods: Magnetic resonance imaging and 3D digital image analysis were used to determine cartilage volume before and after physical activity in the patella of 12 volunteers (knee bends, squatting, normal gait, running, cycling). Deformation of femorotibial cartilage was investigated in 10 subjects (knee bends, static compression, high impact loading). Patellar cartilage deformation after knee bends was compared in seven professional weight lifters, seven sprinters, and 14 untrained volunteers.

Results: Patellar cartilage deformation was $-5.9 \%$ after knee bends, $-4.7 \%$ after squatting, $-2.8 \%$ after normal walking, $-5.0 \%$ after running, and $-4.5 \%$ after cycling. The pattern of patellar cartilage deformation corresponded to the range of motion involved in the particular activity. Tibial cartilage deformation was greatest under high impact loading $(-7 \%)$, but small for other activities. No significant difference was found between athletes and non-athletic controls.

Conclusions: Patellar cartilage deformation shows a "dose dependent" response, where more intense loading leads to greater deformation. Relatively little deformation was observed in the femorotibial joint, except during high impact activities. The findings provide no evidence that adult human cartilage properties are amendable to training effects in vivo.
$\mathrm{K}$ nowledge on the deformational behaviour of articular cartilage in vivo is required in order to understand both the pathogenesis of osteoarthritis and the mechanical target environment of prospective cartilage transplant recipients. However, current knowledge of the mechanical properties and deformational behaviour of articular cartilage has been largely based on in vitro experiments of cartilage samples excised from their natural environment. ${ }^{12}$ These in vitro experiments do not permit the deformational behaviour of cartilage to be estimated in vivo, because the forces across joints are unknown for physiological activities, the load transmission across cartilage layers is complex and highly variable during dynamic activities, and in vitro experiments do not account for the normal (cartilage on cartilage) loading and lubrication conditions of naturally incongruent surfaces.

Quantitative knowledge of in vivo articular cartilage deformational behaviour is, however, useful for a better understanding of cartilage physiology and pathophysiology. Cartilage deformation is dependent on the biochemical composition of the tissue and may represent a more sensitive marker of early osteoarthritis than macro-morphological variables such as cartilage volume, thickness, and joint space narrowing. ${ }^{3}$ Knowledge of magnitudes of strain in the target tissue is important when designing cartilage transplants. In doing so, artificial cartilage can be designed to meet strain requirements ex vivo so that cartilage transplants will also withstand these strains in vivo. The magnitude of in vivo deformation of articular cartilage is related to the magnitude of the mechanical stimulus experienced by the chondrocytes, which is known to affect their biosynthetic activity. ${ }^{4-6}$ Knowledge about in vivo cartilage deformation may thus serve as an important guide to how to stimulate cells optimally in tissue culture and cartilage transplants, and also in cartilage in situ using mechanical signals.

Recently, high resolution magnetic resonance imaging (MRI) and dedicated image analysis tools have been developed to determine cartilage morphology with high precision. $^{78}$ These techniques allow one to examine the deformational behaviour of articular cartilage in vivo. Surface to surface strains across patellar cartilage have been shown to have an average of approximately $5-6 \%$ after knee bends. ${ }^{9} \mathrm{~A}$ period of 90 minutes was subsequently required for patellar cartilage to recover fully from this magnitude of deformation. ${ }^{10}$ Static activity (squatting) involves a smaller amount of deformation than dynamic exercise such as knee bends, ${ }^{11}$ and elderly subjects (without osteoarthritis) have significantly smaller amounts of deformation than young healthy volunteers. $^{12}$ It has also been shown that no significant diurnal changes occur in total femoral cartilage volume, but that there are subtle differences in regional cartilage thickness. ${ }^{13}$

No previous study has examined the deformational behaviour of patellar cartilage under a large variety of physiological activities, such as walking, cycling, and running. Nor has any study addressed the magnitude of femorotibial cartilage deformation after different types of activity, although cartilage properties have been shown to vary among knee joint cartilage plates. ${ }^{14}$ The first objective of this study was thus to explore the magnitude and distribution of patellar cartilage deformation after different types of physiological activity that involve varying degrees of knee motion and load magnitudes in vivo. The second objective was to examine the magnitude of femorotibial cartilage deformation after various types of physiological activity. 


\begin{tabular}{|c|c|c|c|c|c|}
\hline & Patella & MT & LT & MFC & LFC \\
\hline Volume short term & $1.0 \%$ & $2.0 \%$ & $2.2 \%$ & $3.8 \%$ & $4.1 \%$ \\
\hline Volume long term & $1.6 \%$ & $5.4 \%$ & $3.9 \%$ & $4.9 \%$ & $4.1 \%$ \\
\hline Mean thick short term & $1.2 \%$ & $1.6 \%$ & $1.5 \%$ & $2.5 \%$ & $3.1 \%$ \\
\hline Mean thick long term & $2.2 \%$ & $3.2 \%$ & $2.7 \%$ & $5.1 \%$ & $3.0 \%$ \\
\hline
\end{tabular}

LFC, lateral femoral condyle; LT, lateral tibia; MFC, medial femoral condyle; MT, medial tibia.

There is a suggestion from several animal models that cartilage composition and mechanical properties depend on the level of physical training. ${ }^{15}$ Whether or not cartilage microstructure, composition, and mechanical properties are amendable to "training" effects and can functionally adapt to mechanical stimuli in humans is still an open question. The third objective of our study was therefore to test the hypothesis that athletes show less patellar cartilage deformation than non-athletic volunteers, because the mechanical stimulation causes an increase in the metabolic activity of the chondrocytes, the proteoglycan content of the cartilage matrix, and the stiffness of the cartilage.

\section{METHODS}

We examined three samples of healthy volunteers (50 in total), none of whom had a history of symptoms, signs, trauma, or surgery at the knee joint. Informed written consent was obtained from all volunteers and the study protocol was ratified by the local ethic committee.

All magnetic resonance (MR) images were acquired with a clinical 1.5T magnetic resonance scanner (Magnetom Vision, Siemens, Erlangen, Germany).

\section{Magnitude of patellar cartilage deformation after different types of exercise}

To determine the effect of various types of exercise on patellar cartilage deformation, we examined 12 healthy volunteers aged 23 to 30 years (six men and six women). In a first session, four axial scans of the patellar cartilage were acquired after 60 minutes of physical rest using a FLASH water excitation sequence ${ }^{11} 12$ 16-21 (time of repetition $(\mathrm{TR})=17.2 \mathrm{~ms}$, time of echo $(\mathrm{TE})=6.6 \mathrm{~ms}$, flip angle $=20^{\circ}$, bandwidth $=120 \mathrm{~Hz} /$ pixel, slice thickness $=1.5 \mathrm{~mm}$, in-plane resolution $=0.3 \mathrm{~mm} \times 0.3 \mathrm{~mm}$; acquisition time $=3 \mathrm{~min}$ $47 \mathrm{~s})$. The knee was repositioned within the coil in between the four repeated scans. The volunteers were then asked to do 30 deep knee bends, as described previously. ${ }^{11}$ Another axial scan was then acquired starting at 90 seconds after the end of the knee bends. The same volunteers were re-examined over a period of six to 12 months. Upon re-examination one axial scan was acquired before and one after a specific activity. These activities included squatting at a $90^{\circ}$ knee angle for 20 seconds, ${ }^{11}$ normal walking at ground level for five minutes, running $200 \mathrm{~m}$, walking up and down 54 steps over a total time of four minutes, and cycling for 10 minutes on a training bike at $80 \mathrm{~Hz}$ frequency.

\section{Magnitude of femorotibial cartilage deformation after different types of exercise}

To determine the effects of various types of activities on femorotibial cartilage deformation, we examined 10 volunteers aged 18 to 37 years (five men and five women), employing the same sequence as described above but using a coronal section orientation (acquisition time seven minutes). Two coronal scans (with repositioning) were acquired after 60 minutes of rest in a first session, and then one coronal scan after 30 knee bends. The same volunteers were reexamined over a period of six to 12 months, before and after

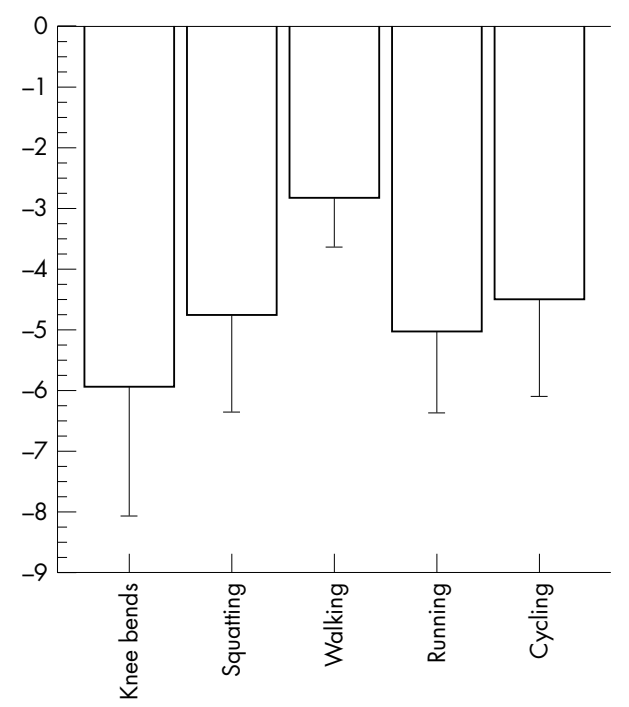

Figure 1 Magnitude of patellar cartilage deformation after knee bends, squatting (20 second static loading at $90^{\circ}$ knee flexion), normal walking, running (including steps), and cycling.

a specific activity. The activities included 30 knee bends done on one leg only, two minutes of static loading of the femorotibial joint of one leg at $15^{\circ}$ flexion with $200 \%$ body weight, and 10 jumps from a chair $(40 \mathrm{~cm}$ height) onto one leg.

\section{Magnitude of patellar cartilage deformation in athletes $v$ non-athletic volunteers}

To determine whether the deformational behaviour of patellar cartilage differed in athletes and in non-athletic controls, we examined the effect of 30 knee bends in 14 men (mean (SD) age, 24.9 (1.8) years) who had never undertaken regular strength training. These men were compared with seven professional weight lifters (age 23.0 (3.2) years) and seven professional bobsleigh sprinters (age 29.3 (4.2) years) who were all currently active. The weightlifters had been at least Bavarian champion and included one world champion. The bobsleigh sprinters had been at least third in a German championship and included three world champions and one Olympic vice-champion.

\section{Digital image analysis of MR imaging data and reproducibility (precision)}

Cartilage volume and thickness were determined after segmentation, ${ }^{22}$ using three dimensional reconstruction and Euclidean distance transformation, ${ }^{23}$ as described previously. ${ }^{11} 121^{17-21}$ The magnitude of cartilage deformation was assessed by subtracting the cartilage volume determined after a specific activity from that obtained before the activity. This value was expressed by the percentage difference from the value before the activity. 


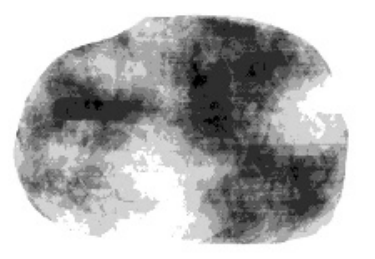

Knee bends
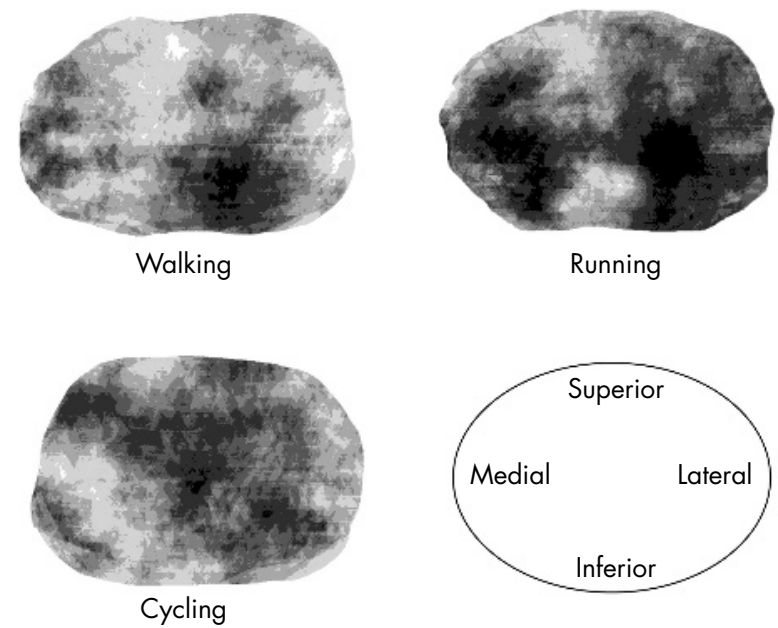

Figure 2 Regional variation of deformation throughout the patella for different types of activities; average over all 12 volunteers. View on the retropatellar surface from posteriorly (lateral facet on the right and medial facet on the left). Dark areas are regions of high deformation and lighter areas are regions of low deformation. The results show that activities with a limited range of knee motion (squatting and walking) lead to deformation of some confined areas, whereas activities that involve a larger range of motion lead to more widespread deformation of patellar cartilage.

To determine the short term precision of the analyses of the various cartilage plates, we computed the root mean square coefficient of variation (RMS CV\%) ${ }^{24}$ of repeated baseline measurements acquired in the first session, with image analysis also being done within a single session. To determine the "long term acquisition/resegmentation" precision of the measurements, we computed the root mean square coefficient of variation (RMS CV\%) ${ }^{24}$ of all baseline measurements acquired over a period of six to 12 months, with image analysis being undertaken in different sessions also spread over a period of six to 12 months. In the latter case, the users were blinded to their previous segmentations.

In the patella, the short term precision error (four repeat scans during the first session; image analysis in one session) was $1.0 \%$ (RMS CV\%) for cartilage volume and $1.2 \%$ for mean cartilage thickness (table 1). The long term acquisition/ resegmentation precision error (five repeat (baseline) scans) was $1.6 \%$ for cartilage volume and $2.2 \%$ for mean cartilage thickness. These errors were random and not systematic, and there was no "drift" in the data (values becoming systematically smaller or larger with time). In the femorotibial joint the short term precision error (two repeat scans during the first session) ranged from $1.5 \%$ (mean cartilage thickness in the lateral tibia) to $3.8 \%$ (cartilage volume in the medial femoral condyle) (table 1). The long term acquisition/ resegmentation precision error (four repeat (baseline) scans) ranged from $2.7 \%$ (mean cartilage thickness in the lateral tibia) to $5.4 \%$ (cartilage volume of the medial tibia), with again no drift being seen in the data (table 1).

To visualise the pattern of cartilage deformation throughout the patella after different activities, we employed the

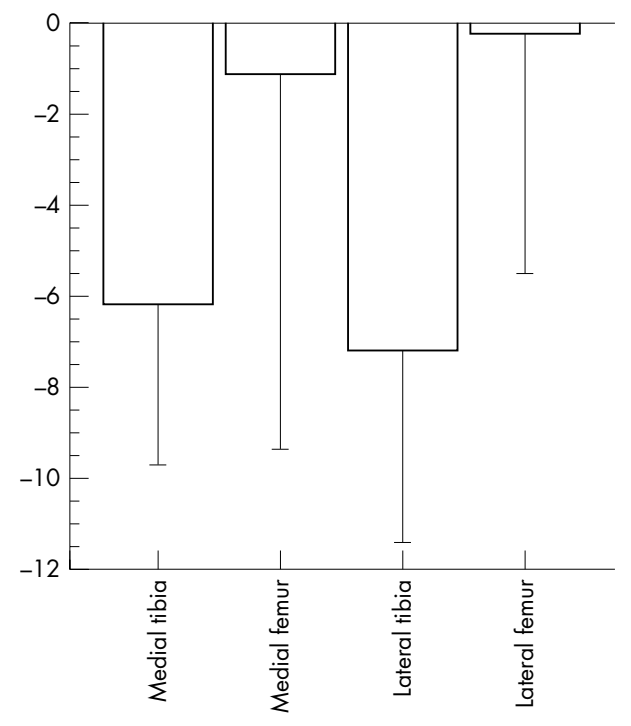

Figure 3 Magnitude of femorotibial cartilage deformation after high impact loading for the medial tibia, lateral tibia, medial femoral condyle, and lateral femoral condyle.

matching algorithm described by Stammberger et al. ${ }^{25}$ Cartilage thickness difference maps for the 12 volunteers were displayed using grey value coding. Averages over the 12 volunteers (for each activity) were derived using commercial software (Adobe Photoshop 7.0, Adobe Systems Inc, San Jose, California, USA).

\section{RESULTS}

The cartilage deformation of the patella (mean (SD) was $-5.9(2.1) \%$ after 30 knee bends, -4.7 ( 1.6$) \%$ after squatting, $-2.8(0.8) \%$ after walking, -5.0 (1.3)\% after running, and -4.5 (1.6)\% after cycling (fig 1). These changes were significant at a $1 \%$ error level. Figure 2 shows the regional variation in deformation throughout the patella for the different activities. Whereas changes were confined to limited regions during squatting and walking, the deformation involved a more widespread area during activities that involved a larger range of knee motion, such as $200 \mathrm{~m}$ running (including 54 stairs), cycling, and knee bends.

In the non-athletic volunteers, the change in patellar cartilage volume after knee bends was $-4.1(2.6) \%$. Changes were $-2.9(1.9) \%$ in the weight lifters and $-3.9(1.8) \%$ in the bobsleigh sprinters. Changes were significant at a $1 \%$ error level in all groups, but differences in deformation between groups were not statistically significant at the $5 \%$ error level.

No significant change in cartilage volume was observed in femorotibial cartilage after two-legged knee bends (except in the lateral tibia) or one-legged knee bends (table 2). Highly significant changes were seen in the medial and lateral tibia after jumps from $40 \mathrm{~cm}$ height, but not in the medial or lateral femoral condyle (fig 3). Only changes of borderline significance were seen in the medial tibia and lateral femoral condyle after the static loading exercise (table 2).

\section{DISCUSSION}

One objective of this study was to determine the magnitude and distribution pattern of patellar cartilage deformation after various physiological activities with different load magnitudes and ranges of motion. The methodology employed to address this question has been shown to be valid $^{1726}$ and reproducible in vivo. ${ }^{17}{ }^{21-23} 25$ Changes (deformation) measured in patellar cartilage were three to six times greater than the precision error (RMS CV\%) of the method, 
Table 2 Cartilage deformation (cartilage volume change) in the femorotibial compartment after various exercises

\begin{tabular}{lllll}
\hline & MT & LT & MFC & LFC \\
\hline Knee bends (2 legs) & $+0.1(4.2) \%$ & $-2.8(4.0) \% * *$ & $-3.9(9.4) \%$ & $-3.3(6.1) \%$ \\
Knee bends (1 leg) & $+0.1(2.4) \%$ & $0.0(2.8) \%$ & $-3.2(8.7) \%$ & $-0.1(4.7) \%$ \\
Jumps (40 cm height) & $-6.1(3.5) \%^{* * *}$ & $-7.2(4.2) \% * *$ & $-1.1(8.3) \%$ & $-0.2(5.2) \%$ \\
Static exercise & $-3.1(4.5) \% *$ & $-2.4(5.2) \%$ & $0.0(6.6) \%$ & $-3.3(6.2) \%^{*}$ \\
\hline Values are mean (SD). & & & \\
*Borderline significance (p<0.1); ** significant at 5\% error level (p<0.05); ${ }^{* * *}$ significant at $1 \%$ error level \\
(p<0.01). \\
LFC, lateral femoral condyle; LT, lateral tibia; MFC, medial femoral condyle; MT, medial tibia.
\end{tabular}

and the knee bend exercise used here produced very similar findings to two previous studies employing the same type of exercise. ${ }^{10}$ Comparing different types of exercise with this technique we found that patellar cartilage shows a "dose dependent" response, more intense loading leading to greater in vivo surface to surface strains. The deformation pattern of the patellar cartilage corresponds to the range of motion involved in the particular activity. Activities with a small range of knee motion cause a confined deformation of relatively small regions of the joint surface, whereas those with a larger range of motion cause a more widespread deformation throughout the joint surface. It is interesting to note that the deformation patterns observed with this technique correspond closely with the patellar contact areas as described by Hehne ${ }^{27}$ for flexion angles of the knee involved in these various activities.

Another objective of this study was to explore femorotibial cartilage deformation after various activities in vivo. Deformation in the femorotibial cartilage was relatively small, even after intense loading. Among the different activities, high impact loading (jumping) caused the largest changes in tibial cartilage, with smaller (insignificant) changes being observed in the femoral condyles. In the femorotibial joint, changes following loading were in the range of the precision error reported for the technique and were thus not consistently significant for different types of exercise and different cartilage plates. The observation that tibial, and particularly femoral, cartilage deforms less than patellar cartilage is consistent with ex vivo measurement of cartilage mechanical properties. ${ }^{14}$ Froimson et al ${ }^{14}$ reported that femoral cartilage showed a $30 \%$ higher compressive aggregate modulus $(\mathrm{p}<0.001)$ and a $66 \%$ lower permeability $(\mathrm{p}<0.001)$ than patellar cartilage. Moreover, the water content of the femur was found to be lower $(5 \%, p=0.031)$ and the proteoglycan content higher $(19 \%, p=0.030)$ than that of the patella. ${ }^{14}$ The investigators hypothesised that the differences in biochemical and mechanical properties between the patella and femur may explain why patellar cartilage shows earlier and more severe fibrillation than the femoral cartilage clinically. Our results confirm that the mechanical properties of cartilage differ among cartilage plates and compartments of the knee, and are associated with the in vivo behaviour of human cartilage. These findings indicate that cartilage transplants with different properties may have to be generated ex vivo to be optimally suited to the mechanical environment of the patella and the femorotibial joint, respectively.

It is tempting to speculate that in vivo cartilage deformation may represent a more responsive marker in early osteoarthritis than macro-morphological variables such as cartilage volume and thickness, because cartilage loss and swelling may occur in parallel in early osteoarthritis. ${ }^{3}$ The technique used here may be employed to detect changes in cartilage composition in early osteoarthritis, or even to monitor the effect of structure modifying osteoarthritis drugs
(SMOADs) in early stages of the disease. High impact loading exercises are inappropriate for patients with osteoarthritis, but the static loading exercise used here may be suitable for investigating patients with early osteoarthritis. As cartilage has been reported to become less stiff and display higher permeability in osteoarthritis, ${ }^{28}$ in vivo deformation of femorotibial cartilage may be greater and become statistically significant in patients. However, this will have to be examined and verified in future studies.

The third objective of our study was to test the hypothesis that the in vivo deformation of patellar cartilage is smaller in athletes than in non-athletic volunteers. This hypothesis was based on the finding that cartilage composition and mechanical properties adapt functionally to mechanical stimulation in animal experiments. ${ }^{15}$ However, in our study no difference in patellar cartilage deformation was observed between athletes and non-athletic volunteers, and at least one previous animal study has shown that training of dogs did not alter the morphological, compositional, or mechanical properties of articular cartilage, even though the animal had been training throughout life. ${ }^{29}$ These different outcomes of animal models may have to do with differences in the maturity of the cartilage, and in susceptibility to mechanical stimuli at different stages of skeletal maturity.

One in vivo study has shown substantial and significant differences in the deformational behaviour of cartilage of young and elderly volunteers, using the same in vivo technique employed here. ${ }^{12}$ These differences have been attributed to differences in cartilage composition, specifically an increase in collagen crosslinks. The results of this study have thus indicated that age dependent changes in cartilage composition and mechanical properties have potential to be measured in vivo. However, our current study provides no evidence that intense physical training modulates cartilage composition to a degree sufficient to allow differences in its deformational behaviour to be detected in vivo. Our findings thus do not provide evidence that normal adult human cartilage mechanical properties are amendable to training effects using mechanical stimulation in vivo.

\section{ACKNOWLEDGEMENTS}

We would like to thank Marcus Tieschky for image acquisition and segmentation in the femorotibial compression experiments. Emily McWalter is acknowledged for English editing This study was supported by a grant from the German Research Foundation (DFG).

\footnotetext{
Authors' affiliations

F Eckstein, M Hudelmaier, Institute of Anatomy and Musculoskeletal Research, Paracelsus Medical Private University, Salzburg, Austria B Lemberger, C Gratzke, Musculoskeletal Research Group, Institute of Anatomy, Ludwig-Maximilians-Universität München, Munich, Germany C Glaser, M Reiser, Institute for Clinical Radiology, Klinikum der LudwigMaximilians-Universität München, Munich

K-H Englmeier, Institute for Medical Informatics and System Research (MEDIS), GSF Forschungszentrum für Umwelt und Gesundheit, Oberschleißheim, Germany
} 


\section{REFERENCES}

1 Mow VC, Ateshian GA, Spilker RL. Biomechanics of diarthrodial joints: a review of twenty years of progress. J Biomech Eng 1993;115:460-7.

2 Mow VC, Holmes MH, Lai WM. Fluid transport and mechanical properties of articular cartilage: a review. J Biomech 1984;17:377-94.

3 Burstein D, Bashir A, Gray ML. MRI techniques in early stages of cartilage disease. Invest Radiol 2000;35:622-38.

4 Sah RL, Kim YJ, Doong JY, Grodzinsky AJ, Plaas AH, Sandy JD. Biosynthetic response of cartilage explants to dynamic compression. J Orthop Res 1989:7:619-36.

5 Kim YJ, Bonassar L, Grodzinsky AJ. The role of cartilage streaming potential, fluid flow and pressure in the stimulation of chondrocyte biosynthesis during dynamic compression. J Biomech 1995;28:1055-66.

6 Urban JP. The chondrocyte: a cell under pressure. $\mathrm{Br} J$ Rheumatol 1994;33:901-8.

7 Peterfy CG, Genant HK. Emerging applications of magnetic resonance imaging in the evaluation of articular cartilage. Radiol Clin North Am 1996;34:195-213.

8 Eckstein F, Reiser M, Englmeier KH, Putz R. In vivo morphometry and functional analysis of human articular cartilage with quantitative magnetic resonance imaging - from image to data, from data to theory. Anat Embryol (Berl) $2001 ; 203: 147-73$

9 Eckstein F, Tieschky M, Faber SC, Haubner M, Kolem H, Englmeier KH, et al. Effect of physical exercise on cartilage volume and thickness in vivo: MR imaging study. Radiology 1998;207:243-8.

10 Eckstein F, Tieschky M, Faber S, Englmeier KH, Reiser M. Functional analysis of articular cartilage deformation, recovery, and fluid flow following dynamic exercise in vivo. Anat Embryol (Berl) 1999;200:419-24.

11 Eckstein F, Lemberger B, Stammberger T, Englmeier KH, Reiser M. Patellar cartilage deformation in vivo after static versus dynamic loading. J Biomech 2000;33:819-25

12 Hudelmaier M, Glaser C, Hohe J, Englmeier KH, Reiser M, Putz R, et al. Agerelated changes in the morphology and deformational behavior of knee joint cartilage. Arthritis Rheum 2001;44:2556-61.

13 Waterton JC, Solloway S, Foster JE, Keen MC, Gandy S, Middleton BJ, et al. Diurnal variation in the femoral articular cartilage of the knee in young adult humans. Magn Reson Med 2000;43:126-32.

14 Froimson MI, Ratcliffe A, Gardner TR, Mow VC. Differences in patellofemoral joint cartilage material properties and their significance to the etiology of cartilage surface fibrillation. Osteoarthritis Cartilage 1997;5:377-86.

15 Vanwanseele B, Lucchinietti E, Stussi E. The effects of immobilization on the characteristics of articular cartilage: current concepts and future directions. Osteoarthritis Cartilage 2002;10:408-19.

16 Hyhlik-Dürr A, Faber S, Burgkart R, Stammberger T, Maag KP, Englmeier KH, et al. Precision of tibial cartilage morphometry with a coronal water-excitation MR sequence. Eur Radiol 2000;10:297-303.
17 Burgkart R, Glaser C, Hyhlik-Durr A, Englmeier KH, Reiser M, Eckstein F. Magnetic resonance imaging-based assessment of cartilage loss in severe osteoarthritis: accuracy, precision, and diagnostic value. Arthritis Rheum 2001;44:2072-7.

18 Eckstein F, Heudorfer L, Faber SC, Burgkart R, Englmeier KH, Reiser M. Longterm and resegmentation precision of quantitative cartilage $M R$ imaging (qMRI). Osteoarthritis Cartilage 2002;10:922-8.

19 Vanwanseele B, Eckstein F, Knecht H, Stussi E, Spaepen A. Knee cartilage of spinal cord-injured patients displays progressive thinning in the absence of normal joint loading and movement. Arthritis Rheum 2002;46:2073-8.

20 Vanwanseele B, Eckstein F, Knecht H, Spaepen A, Stüssi E. Longitudinal analysis of cartilage atrophy in the knees of spinal cord injured patients. Arthritis Rheum 2003;48:3377-81.

21 Glaser C, Burgkart R, Kutschera A, Englmeier KH, Reiser M, Eckstein F. Femoro-tibial cartilage metrics from coronal MR image data: technique, test-retest reproducibility and findings in OA. Magn Reson Med 2003;50:1229-36.

22 Stammberger T, Eckstein F, Michaelis M, Englmeier KH, Reiser M Interobserver reproducibility of quantitative cartilage measurements: comparison of B-spline snakes and manual segmentation. Magn Reson Imaging 1999;17:1033-42.

23 Stammberger T, Eckstein F, Englmeier KH, Reiser M. Determination of 3D cartilage thickness data from MR imaging: computational method and reproducibility in the living. Magn Reson Med 1999;41:529-36.

24 Glüer CC, Blake G, Lu Y, Blunt BA, Jergas M, Genant HK. Accurate assessment of precision errors: how to measure the reproducibility of bone densitometry techniques. Osteoporos Int 1995;5:262-70.

25 Stammberger T, Hohe J, Englmeier KH, Reiser M, Eckstein F. Elastic registration of $3 D$ cartilage surfaces from MR image data for detecting local changes in cartilage thickness. Magn Reson Med 2000;44:592-601.

26 Graichen H, von Eisenhart-Rothe R, Vogl T, Englmeier KH, Eckstein F. Quantitative assessment of cartilage status in osteoarthritis by quantitative magnetic resonance imaging: technical validation for use in analysis of cartilage volume and further morphologic parameters. Arthritis Rheum 2004;50:811-16.

27 Hehne HJ. Biomechanics of the patellofemoral joint and its clinical relevance. Clin Orthop 1990:73-85.

28 Armstrong CG, Mow VC. Variations in the intrinsic mechanical properties of human articular cartilage with age, degeneration, and water content. J Bone Joint Surg Am 1982;64:88-94.

29 Newton PM, Mow VC, Gardner TR, Buckwalter JA, Albright JP. The effect of lifelong exercise on canine articular cartilage. Am J Sports Med 1997;25:282-7. 